\title{
Progress of the ELISE test facility: towards one hour pulses in hydrogen
}

\author{
D. Wünderlich, U. Fantz, B. Heinemann, W. Kraus, R. Riedl, C. Wimmer and the NNBI team
}

Max-Planck-Institut für Plasmaphysik, Boltzmannstraße 2, 85748 Garching, Germany

\begin{abstract}
In order to fulfil the ITER requirements, the negative hydrogen ion source used for NBI has to deliver a high source performance, i.e. a high extracted negative ion current and simultaneously a low coextracted electron current over a pulse length up to one hour. Negative ions will be generated by the surface process in a low-temperature low-pressure hydrogen or deuterium plasma. Therefore, a certain amount of caesium has to be deposited on the plasma grid in order to obtain a low surface work function and consequently a high negative ion production yield. This caesium is re-distributed by the influence of the plasma, resulting in temporal instabilities of the extracted negative ion current and the co-extracted electrons over long pulses. This paper describes experiments performed in hydrogen operation at the half-ITER-size NNBI test facility ELISE in order to develop a caesium conditioning technique for more stable long pulses at an ITER relevant filling pressure of $0.3 \mathrm{~Pa}$. A significant improvement of the long pulse stability is achieved. Together with different plasma diagnostics it is demonstrated that this improvement is correlated to the interplay of very small variations of parameters like the electrostatic potential and the particle densities close to the extraction system.
\end{abstract}

\section{Introduction}

The neutral beam injection (NBI) system at ITER will be used for heating the tokamak plasma and for current drive [1,2]. An essential part of the NBI beam line is the negative hydrogen ion source, capable of delivering an extracted current of $57 \mathrm{~A}$ for $3600 \mathrm{~s}$ in deuterium operation and $66 \mathrm{~A}$ for $1000 \mathrm{~s}$ in hydrogen (corresponding to extracted current densities of $28.5 \mathrm{~mA} / \mathrm{cm}^{2}$ and $33.0 \mathrm{~mA} / \mathrm{cm}^{2}$, respectively).

In order to minimize the destruction rate of negative ions in the accelerator and to additionally limit the power loads in the extraction system, the source has to be operated at a filling pressure of $\leq 0.3 \mathrm{~Pa}$. The amount of co-extracted electrons must be equal or smaller than the amount of extracted negative ions. In order to ensure good beam optics, hence good beam transmission, deviations in the uniformity of the extracted beam of $<10 \%$ are required.

ELISE (Extraction from a Large Ion Source Experiment) $[3,4]$ is part of a R\&D roadmap

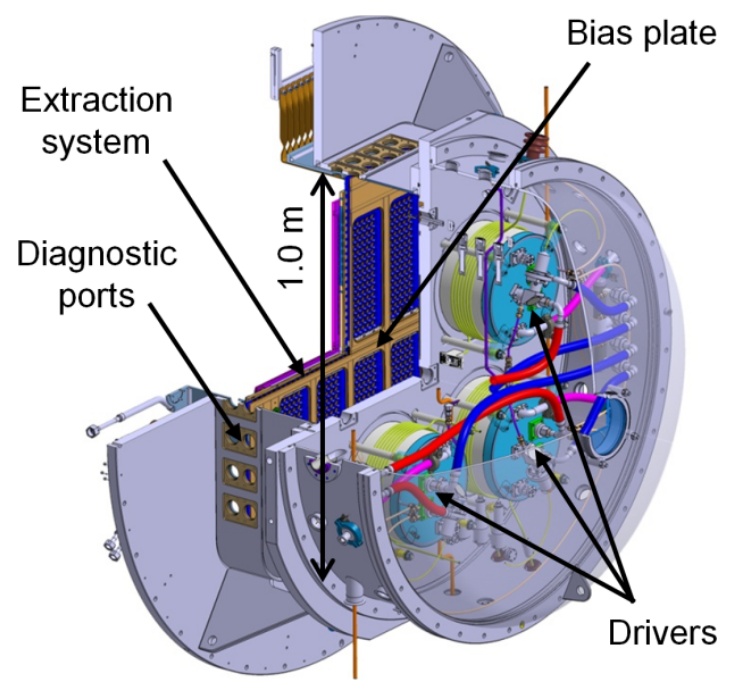

Figure 1: Schematic view of the ELISE ion source. The plasma is generated in the four drivers and then expands towards the extraction system. 
defined by the European domestic agency F4E for the construction of the neutral beam heating systems $[5,6]$ : the half-ITER-size ion source of the ELISE test facility $\left(0.9 \times 1.0 \mathrm{~m}^{2}\right.$ with an extraction area of $\left.0.1 \mathrm{~m}^{2}\right)$ is an intermediate step between the RF driven ITER prototype source $\left(0.3 \times 0.6 \mathrm{~m}^{2}\right.$ with an extraction area of typically $\left.6 \cdot 10^{-3} \mathrm{~m}^{2}\right)$ [7] and the ion source for the ITER NBI system $\left(1.0 \times 2.0 \mathrm{~m}^{2}\right.$ with an extraction area of $0.2 \mathrm{~m}^{2}$ ) [8]. The latter is in principle identical with the ion source used at the SPIDER and MITICA test facilities under construction at the neutral Beam Test Facility PRIMA in Padova $[9,10]$.

Figure 1 shows a schematic view of the ELISE ion source. The design of the ion source and the extraction system is as close as possible to the ITER design but differs in some details in order to enable better diagnostic access and more experimental flexibility [3]. ELISE is designed for cw plasma operation. However, due to restrictions in the available power supplies short beam blips (length: $10 \mathrm{~s}$ each, extraction voltage up to $15 \mathrm{kV}$, total voltage up to $60 \mathrm{kV}$ ) are possible each $\approx 150 \mathrm{~s}$ only. During one operational day up to around hundred plasma pulses can be performed (the number depending on the pulse length); the pause time between the plasma pulses typically several minutes.

ELISE can be operated in hydrogen or in deuterium. The plasma is generated by inductive RF coupling $\left(\mathrm{P}_{\mathrm{RF}}<90 \mathrm{~kW} /\right.$ driver $)$ in the four cylindrical drivers and then expands into the rectangular expansion region (horizontal and vertical dimension $\approx 1 \mathrm{~m}$, axial dimension $\approx 0.27 \mathrm{~m}$ ). In order to minimize the amount of co-extracted electrons, a magnetic filter field created by a current flowing through the PG $\left(I_{P G}\right)$ is used ( $\mathrm{I}_{\mathrm{PG}}$ is usually $<4 \mathrm{kA}$ ) [11] and additionally a positive bias potential is applied to the PG with respect to the source body being connected to the so-called bias plate. The interplay of the magnetic filter field and the PG bias can result in vertical plasma drifts [12].

The majority of negative hydrogen ions are produced via the surface process [13]: hydrogen atoms or positive hydrogen ions pick up electrons from the inner surfaces of the source and are reflected towards the bulk plasma as negative ions. The efficiency of this conversion process is drastically increased by a thin layer of caesium, which decreases the surface work function [14]. The cesium layers in ELISE are significantly thicker (several monolayers) than the 0.6 monolayers for which an optimum work function $(\Phi=1.4 \mathrm{eV}$ without the presence of impurities) would be expected. The work function (for the parameters of ELISE typically around $2.2 \mathrm{eV}$ [15]) is not a function of the thickness of the caesium layer. Caesiating the source surfaces additionally results in a strong reduction of the co-extracted electron current [7].

Due to a short survival length of the negative ions (in the $\mathrm{cm}$ range in the drivers, up to a few tens of $\mathrm{cm}$ in close proximity to the extraction system [16]), the most relevant converter is the surface of the plasma grid (PG), the first grid of the extraction system consisting of three grids (the PG, the extraction grid and the grounded grid, each comprising 640 apertures, $\varnothing 14 \mathrm{~mm}$ in the PG). The co-extracted electrons are removed from the beam and dumped onto the surface of the extraction grid by the magnetic field created by deflection magnets embedded into the extraction grid [3]. The maximum possible amount of co-extracted electrons is defined by the upper limit of the allowed power deposited onto the extraction grid. Due to this limit a too high amount of co-extracted electrons can restrict operational parameters like the RF power or the extraction voltage and thus the amount of extracted negative ions or the length of a pulse. 
The surface work function at the PG can deteriorate by reactions with impurities embedded into, or deposited on, the caesium layer (from the background gas or the plasma). This deterioration is the main factor causing non-reproducibility between pulses and temporal instabilities during pulses. To fulfil the ITER requirements for the NBI source, the caesium flow onto the PG should be sufficient to counteract these deteriorating effects and ensure a low work function at the PG that is homogeneous over the area of the PG and stable over pulses up to one hour. Caesium is evaporated into ELISE by means of two caesium ovens, attached to the two vertical side walls of the ion source [17]. The caesium from the ovens is re-distributed, mainly by the influence of the plasma [18] and forms reservoirs on the source surfaces. The caesium flow onto the PG is correlated with the position and the capacity of these caesium reservoirs as well as the kind and amount of impurities embedded into or deposited on the reservoirs [18].

For obtaining a high performance a series of dedicated caesium conditioning pulses is necessary. During these pulses the source is operated in a way that improves the subsequent source performance, such as increased stability and/or higher extracted ion currents and/or lower co-extracted electron currents. It is assumed that during this conditioning procedure caesium is redistributed to create suitable reservoirs inside the source. While the procedures related to caesium conditioning for short plasma pulses (plasma pulse length: several seconds) were investigated in detail at the small prototype source $[19,20]$, significantly less experiments regarding long pulses (plasma pulse length: up to several hundreds of seconds) have been done at the prototype source $[20,21]$ and at ELISE [22].

Previously, for low RF power ( $P_{R F}=20 \mathrm{~kW} /$ driver) stable pulses up to a plasma pulse length of $450 \mathrm{~s}$ (four beam blips) have been demonstrated at ELISE in hydrogen with an electron-ion-ratio well below one but only a small extracted negative ion current density of below $10 \mathrm{~mA} / \mathrm{cm}^{2}$ [22]. Most measures increasing the extracted negative ion current (namely increasing the RF power [21], increasing the extraction voltage and decreasing the filter field strength) can result in a higher temporal instability during long pulses, mainly a more pronounced increase with time of the co-extracted electrons. This effect is correlated with the influence of the source and plasma parameters on the caesium reservoirs and the caesium flow towards the PG and consequently the PG work function during the plasma pulses

This paper presents investigations on how to prepare large area NNBI sources like ELISE during the caesium conditioning phase in order to counteract the increase of the co-extracted electrons. The aim is to enable stable pulses with a longer pulse length (up to one hour, i.e. one hour plasma with $10 \mathrm{~s}$ extraction each $180 \mathrm{~s}$ ) and/or a higher performance compared to the best pulses up to now. These experiments have been performed in hydrogen and they were accompanied by beam and plasma diagnostics.

\section{Diagnostics of the beam and the plasma}

The most important diagnostics used at ELISE are electric current measurements [23]: a) the extracted negative ion current (the current to the grounded grid and all beamline components, including the calorimeter); b) the co-extracted electron current (the current to the extraction grid). The extraction grid is divided into two vertical segments that are insulated from each other. Thus, the co-extracted electron current can be measured separately for the upper and the lower part of the beam; c) the drain current 
(the total current flowing back to the HV power supply, i.e. the sum of negative ion and electron currents). The drain current is measured independently from the negative ion current and the coextracted electron current. Additional measurements exist for the current drawn onto the grid holder boxes, the bias current and the current drawn onto the bias plate (distinguishing between the upper and lower part of the bias plate). A detailed description of the electric current measurements at ELISE (including a scheme of the HV circuit with the current measurements) can be found elsewhere [23].

The properties of the beam are determined by calorimetric and spectroscopic measurements [24]. $3.5 \mathrm{~m}$ downstream of the grounded grid a diagnostic calorimeter is installed which intercepts the beam. It consists an array of 30x30 copper blocks with a very low transversal thermal conductivity between the blocks. The beam power and its distribution are diagnosed by means of water flow calorimetry (independent measurements for the four beam sectors [24]), thermocouples and an IR camera. Another beam diagnostic, a tungsten wire calorimeter, is installed $1.8 \mathrm{~m}$ downstream of the grounded grid. It consists of a rectangular array of tungsten wires that are heated by the beam. The wires are observed by a standard camera, which allows a rapid, qualitative, assessment of the beam shape and divergence. Furthermore a beam emission spectroscopy (BES) system is used, which consists of 16 horizontal and 4 vertical lines of sight. The BES system is connected via optical fibers to a high resolution spectrometer and allows the determination of the vertical and horizontal profiles of beam properties like the intensity, the divergence, and the stripping losses, i.e. the amount of negative ion loss by collisions with the background gas in the extraction and acceleration system.

Most of the processes relevant for the production of negative hydrogen ions and for their transport towards the extraction apertures take place in the extended boundary layer (the plasma volume in direct vicinity of the PG with an axial extent of a few $\mathrm{cm}$ ), and therefore the focus of the plasma diagnostics in ELISE is on this plasma volume. The main plasma diagnostic is optical emission spectroscopy (OES) [25]. Lens heads collect the light emitted along several lines-of-sight (LOS) (most of them parallel to the PG and at $3.4 \mathrm{~cm}$ to the PG) and couple it into optical fibers. These fibers are connected to a set of 13 spectrometers. The optical systems are absolutely calibrated. Figure 2 gives a view onto the ELISE extraction system and the lines of sight currently used for OES (red) in order to measure the radiation emitted in the extended boundary layer.

OES is a very simple and powerful technique [26]. However, the evaluation of OES results can be quite complex, especially in plasmas that are in the transition between ionizing and recombining plasmas. This is the case for the plasma of the extended boundary layer [25].

In order to monitor the caesium emission during the conditioning phase, four light sensitive diodes are available, connected via optical fibers to the horizontal LOS XL-2Uu and XL+2Uu and the vertical LOS YB$3 \mathrm{Uu}$ and $\mathrm{YT}+3 \mathrm{Uu}$. Each of these diodes detects light in the visible wavelength range and is combined with an interference filter for the emission line of neutral caesium at $852 \mathrm{~nm}$. The used LOS are depicted in blue in Figure 2. No relative or absolute calibration of the four diodes has been done. However, since identical diodes and identical fibers have been used, the ratio of the emissivity measured along the two LOS gives a rough estimation for the ratio of the emissivity of the $\mathrm{CS}_{852}$ line. Similar as for OES, an 


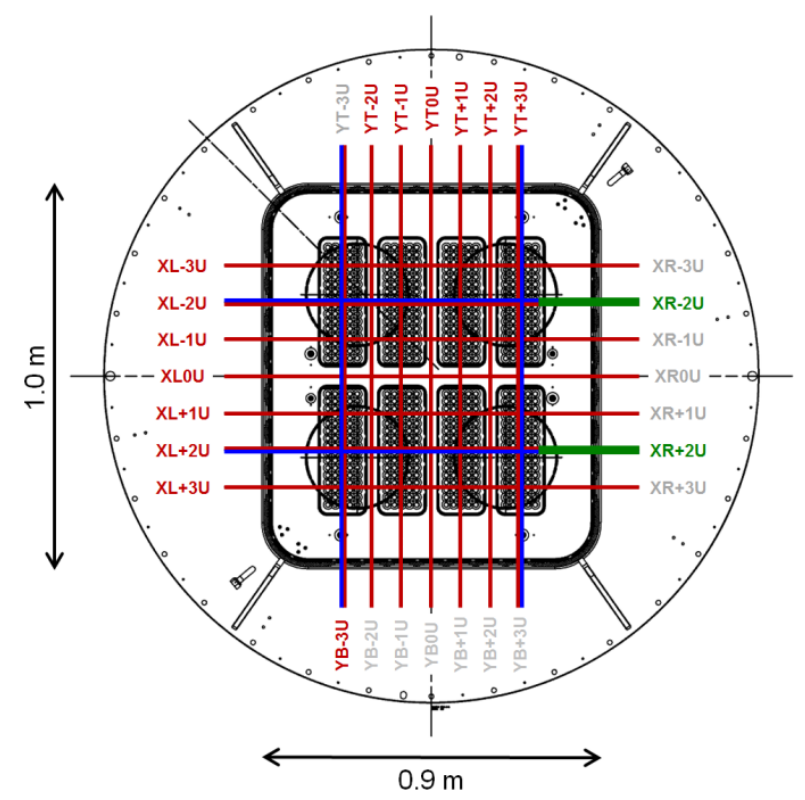

Figure 2: Axial view onto the ELISE extraction system (from the upstream side, i.e. from the plasma). Shown are the diagnostic ports enabling access to the plasma of the extended boundary layer. Ports (and the corresponding LOS) used in the current setup for OES and the Cs diodes are shown in red and blue, respectively. The position of the two Langmuir probes is shown in green. The four circles depict the projection of the four drivers onto the $P G$. here. absolute determination of the caesium density in the extended boundary layer can be complex, especially since in such ion sources caesium is ionized to a large degree [27].

Additionally two fixed Langmuir double probes have been introduced at the diagnostic ports XR$2 U$ and $X R+2 U$ (indicated in green in Figure 2). The probe tips are located close to the right side of the source and in vertical direction close to the projection of the centers of the upper (top probe) and lower (bottom probe) drivers. This setup allows assessing the vertical homogeneity of plasma parameters.

For the measurements presented here, the probes have been operated as single probes i.e. using only one tip of each probe. Thus, the probes allow a standard determination of plasma parameters like the plasma potential, the positive ion density and the electron temperature. Since the probes are not RF compensated, the error bars of the absolute values for the plasma potential and of the electron temperature are quite large. Only relative changes of the potentials are presented

\section{Cs conditioning for long pulses and best results obtained}

Aim of the experiments described in the following is to improve the insight in the correlation of the caesium conditioning process with the source parameters. Pulses have been performed with an already well caesium conditioned source, i.e. a good obtained source performance during short pulses. The caesium ovens were switched on during all pulses, i.e. fresh caesium was steadily evaporated into the source.

The strength of the magnetic filter is an important parameter affecting the source performance during short pulses $[22,16]$. In order to investigate the influence of the filter field strength on the stability of long pulses, a variation of $\mathrm{I}_{\mathrm{PG}}$ between $1.8 \mathrm{kA}$ and $4.2 \mathrm{kA}$ (corresponding to a filter field strength between $1.7 \mathrm{mT}$ and $4.0 \mathrm{mT}$ at the center of the PG) was performed at a filling pressure of $0.35 \mathrm{~Pa}$ and an $\mathrm{RF}$ power of $30 \mathrm{~kW} /$ driver. The extraction voltage was $5 \mathrm{kV}$ and the bias current $55 \mathrm{~A}$. By keeping the bias current constant during pulses - instead of the bias potential - the net charged particle flux onto the PG is kept constant (to within $\pm 0.05 \mathrm{~A}$ during short pulses). It was demonstrated at the prototype source that this operation scenario is beneficial for obtaining stable operation $[7,28]$. 
The $I_{P G}$ variation consists of several long pulses with a plasma pulse length of $170 \mathrm{~s}$ and two beam blips with $10 \mathrm{~s}$ length each. In order to obtain a comparable caesium conditioning status for these long pulses, a conditioning phase consisting of approximately ten short pulses at $\mathrm{p}_{\text {fill }}=0.8 \mathrm{~Pa}$ (increasing the filling pressure is beneficial for the caesium conditioning procedure [22]) with a plasma pulse length of $20 \mathrm{~s}$ and one beam blip was done before each long pulse until stable and reproducible conditions have been reached.

Figure 3 shows for six values of $I_{P G}$ the extracted negative ion current density $j_{\mathrm{ex}}$ versus the coextracted electron current density $\mathrm{j}_{\mathrm{e}}$. Shown in the figure is one data point per beam blip (averaged over the second half of the beam blip), i.e. two points per plasma pulse. The two data points of each of the plasma pulses are interconnected by dashed lines. Additionally shown by single grey symbols are the results of the short conditioning pulses (one value per plasma pulse). The shaded area symbolizes results with an electron-ion-ratio above one. With one exception (second beam blip at $I_{P G}=1.8 \mathrm{kA}$ ) all results fulfill the ITER requirement regarding the electron-ion ratio.

The basic behavior of the source performance during the first beam blip is well known from short pulses performed at ELISE during previous investigations [22]: in the investigated range of $I_{P G}$ the ion current decreases steadily with increasing filter field strength while the electron current first decreases and then saturates (for $I_{P G} \geq 3.2 \mathrm{kA}$ ). The temporal stability of both the extracted ions and the co-extracted electrons is significantly improved by increasing the filter field strength. The choice of the magnetic field strength used during long pulse operation of caesiated negative hydrogen ion sources should result from a compromise between high performance and good temporal stability.

For $I_{P G}=2.2 \mathrm{kA}$ results of two different plasma pulses are shown in Figure 3: the first of these pulses was performed at the beginning of the $I_{P G}$ variation and the second one (emphasized in the figure by an ellipse) was the very last pulse of the scan. This pulse was intended as a reproducibility test. The results of the first beam blip of these two pulses differ only slightly. However, the performance of the second beam blip of the reproducibility pulse is much better, i.e. the temporal stability during the second plasma pulse is higher. This result is quite surprising since a short pulse conditioning was performed prior to each of the long pulses until stable and reproducible conditions have been reached. 
This observation indicates that an apparently stable status of the caesium conditioning for long pulses can be further improved by performing one or more long pulses with low filling pressure and then continuing the conditioning process. Although the source is de-conditioned during the long pulses, the modified caesium distribution caused by the longer interaction of the caesium reservoirs with a plasma at a reduced filling pressure has an advantageous long-term effect.

This caesium conditioning technique was applied in order to prepare ELISE for long plasma pulses with more than two beam blips. Time traces of the extracted ion current density jex and the co-extracted electron current density $j_{\mathrm{e}}$ for the best obtained pulse with an ITER relevant filling pressure $\left(p_{\text {fill }}=0.3 \mathrm{~Pa}\right)$ and for $\mathrm{P}_{\mathrm{RF}}=45 \mathrm{~kW}$ per driver are shown in Figure $4 \mathrm{a}$ ). The bias current is $20 \mathrm{~A}$ and the plasma grid current is $2.8 \mathrm{kA}$. The length of the plasma pulse is $400 \mathrm{~s}$, with three beam blips (length of the beam blips: ten seconds each). The extraction voltage is $7 \mathrm{kV}$, ensuring optimum perveance conditions, i.e. a low beam divergence.
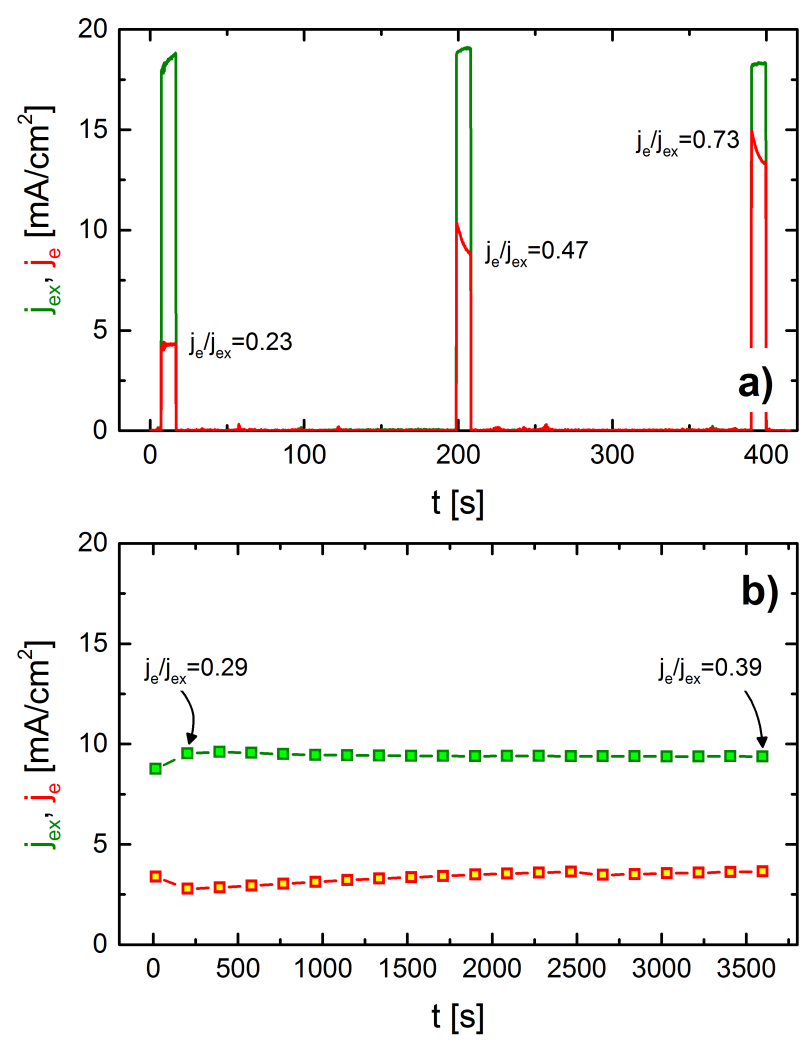

Figure 4: Extracted negative hydrogen ion current density and co-extracted electron current density for the best pulses obtained at ELISE so far at $p_{\text {fill }}=0.3 \mathrm{~Pa}$. (a) $P_{R F}=45 \mathrm{~kW}$ per driver, plasma pulse length $=400 \mathrm{~s}$, three beam blips. (b) $P_{R F}=20 \mathrm{~kW}$ per driver, plasma pulse length $=3600 \mathrm{~s}, 20$ beam blips. Shown for the one hour pulse are results averaged over the second half of the beam blips.
For all three beam blips the ion current density is above $18 \mathrm{~mA} / \mathrm{cm}^{2}$, with a significantly smaller electron current density. While the extracted ion currents measured during the beam blips are comparable, the co-extracted electron current increases strongly from blip to blip but shows a strong decrease during the second and the third beam blip (i.e. a caesium conditioning process takes place during these blips). These results indicate that likely - in contrast to the statement made in [29] - results obtained during pulsed extraction are not fully comparable to results obtained during cw extraction, especially regarding the co-extracted electron current. The most probable reason for this effect is the increased release of caesium from reservoirs at the source back plate during the beam blips caused by backstreaming positive ions from the extraction system [18].

Several pulses of up to one hour plasma pulse length (20 beam blips) have been performed at $\mathrm{p}_{\text {fill }}=0.3 \mathrm{~Pa}$ and $\mathrm{P}_{\mathrm{RF}}=20 \mathrm{~kW}$ per driver. For these pulses a reduced RF power was used in order to increase the stability of the extracted currents. Again, the bias current was set to $20 \mathrm{~A}$ and the plasma grid current to $2.8 \mathrm{kA}$. Figure $4 \mathrm{~b}$ ) shows the extracted ion current density and the coextracted electron current density for the best of 
these pulses. For the sake of clarity, in contrast to Figure 4a) not the complete time traces are shown but averaged values of the extracted currents measured during the beam blips.

Between the first and the second beam blip a distinct improvement of the source performance can been seen. Reason is that this pulse was the second pulse of an operating day, i.e. it was not preceded by the usually performed daily caesium conditioning process. Instead, the caesium conditioning took place during the first minutes of the long plasma pulse. After the second beam blip the extracted negative ion current density is more or less stable (around $9.4 \mathrm{~mA} / \mathrm{cm}^{2}$ ) while for the co-extracted electrons a slight increase is observed (around $30 \%$ ).

Concluding, up to now $50 \%$ of the ITER requirement regarding the extracted negative hydrogen ion current density has been achieved at $\mathrm{p}_{\text {fill }}=0.3 \mathrm{~Pa}$, for a plasma pulse length of $400 \mathrm{~s}$, an electron to ion ratio below one and for less than $50 \%$ of the RF power per driver available at the ITER NBI system (100 kW). Additionally, $25 \%$ of the requirement has been obtained over one hour with $25 \%$ of the RF power available at ITER per driver.

For obtaining these results the described long pulse caesium conditioning technique was essential. However, the strong temporal dependencies observed mainly for the co-extracted electrons at $\mathrm{P}_{\mathrm{RF}}=45 \mathrm{~kW}$ per driver indicate that for completely fulfilling the ITER requirements an even better conditioning status is necessary. Obtaining such a conditioning status is strongly correlated with a better understanding of the correlation of the long pulse conditioning with the plasma parameters.

\section{Correlation with the plasma parameters}

In order to correlate the performed long pulse caesium conditioning procedure with the plasma parameters, a series of four long pulses with identical source parameters have been performed (plasma pulse length $170 \mathrm{~s}$, two beam blips with $10 \mathrm{~s}$ length each). The source parameters are identical to the ones of the pulses in the $I_{P G}$ variation described in the previous section; but the magnetic field strength was kept at a constant value of $I_{P G}=2.2 \mathrm{kA}$. In between the long plasma pulses short conditioning pulses have been performed at an elevated filling pressure until a stable source performance has been reached. Figure 5 shows time traces of different signals measured during the four long plasma pulses.

Shown in Figure 5a) are in full lines time traces of the extracted negative ion current density and the coextracted electron current density. The interconnecting dashed lines act as a guide for the eye and indicate the change of the signals between the two beam blips. The stability of the extracted ion current density and the co-extracted electrons improve from pulse to pulse. Mainly the co-extracted electrons in the second beam blip react strongly on the progress of the caesium conditioning. For the last long pulse of the series almost stable conditions are reached. The electron currents measured separately for both segments of the extraction grid (not shown here) show that the reduction of the electrons is roughly comparable for the upper and lower segment, with a slightly stronger reduction in the bottom part. 
Figure $5 \mathrm{~b}$ ) shows in full lines time traces of the $\mathrm{Cs}_{852}$ emission measured during the four performed long pulses by two of the caesium diodes (upper LOS: XL-2Uu, lower LOS: XL+2Uu, see Figure 2). For all pulses the caesium signal along the lower LOS is higher compared to the upper LOS. Due to the missing calibration of these LOS and the presence of different excitation channels in the recombining plasma of the boundary layer this result does not allow deducing an absolute value for the top/bottom ratio of the total caesium density. However, due to the comparable configuration of the two diodes it indicates that more caesium is present in the lower part of the ion source plasma.

The caesium time traces show the same general behavior for both LOS and for all pulses: a continuous slope with two phases of increased emission during the two beam blips, correlated with the increased caesium release from the back plate caused by the backstreaming ions. It is not clear up to now if this additional caesium release has an advantageous effect on the stability of the source performance during long pulses or not. A short spike of the cesium signal can be seen for the lower LOS at the beginning of the four pulses. This spike is correlated to a gas puff $\left(\mathrm{p}_{\mathrm{fill}} \approx 1.2 \mathrm{~Pa}\right)$ which is necessary for plasma ignition (in combination with two tungsten filaments).

The horizontal dashed lines in Figure $5 b$ ) indicate for both LOS the minimum caesium emission measured during the four pulses and are intended as assistance for assessing the impact of the caesium

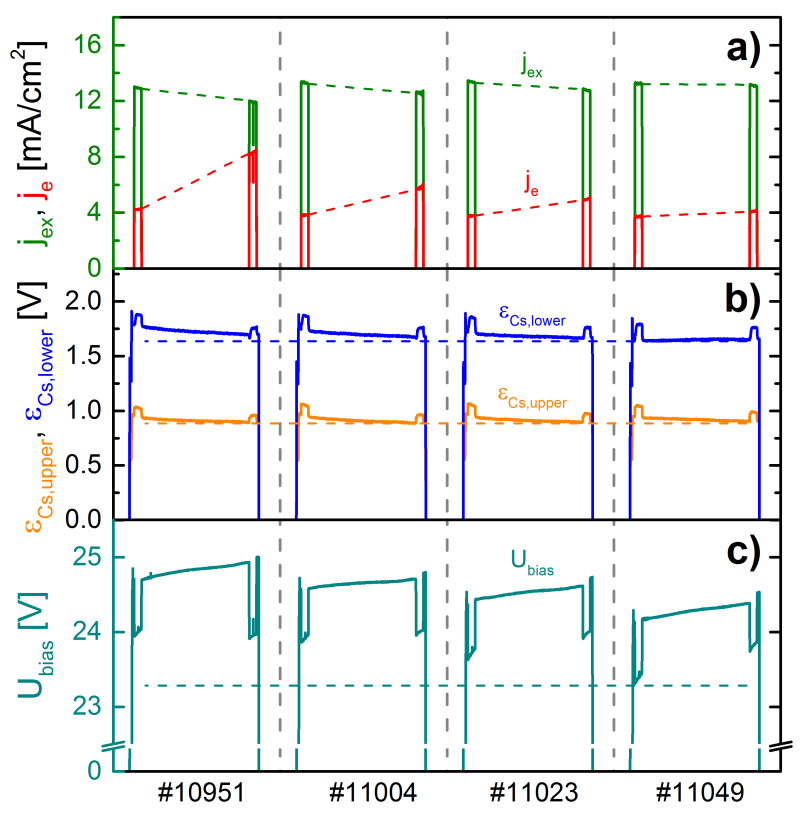

Figure 5: Temporal dependence of different signals taking during the source conditioning process for long pulses ( $t_{\text {plasma }}=170 \mathrm{~s}$ ) with two beam blips at $P_{R F}=30 \mathrm{~kW} /$ driver, $p_{\text {fill }}=0.35 \mathrm{~Pa}$ and $I_{P G}=2.2 \mathrm{kA}$. a) Current density of the extracted ions and coextracted electrons. b) Caesium emissivity measured along the upper and the lower horizontal LOS. c) Bias potential. conditioning process on the temporal behavior of the caesium emission: while the $\mathrm{Cs}_{852}$ emission along the upper LOS is almost not affected by the long pulse conditioning, along the lower LOS both a reduction of the absolute signal and a flattening of the temporal profile is seen. Although this effect is small, it indicates that a more stable source performance is correlated with more stable time traces of the Cs emission.

Shown in Figure $5 c$ ) is the bias potential $U_{\text {bias }}$ measured during the four pulses. The full line represents the measured potential and the horizontal dashed line the lowest value of the bias potential measured during the four long pulses (during the first beam blip of pulse \#11049). The general temporal behavior of $\mathrm{U}_{\text {bias }}$ within single pulses (the potential increases slightly during the plasma pulse, additionally the potential is significantly decreased by the influence of the extraction voltage during the two beam blips) is not altered during the conditioning process. The relative decrease of the bias potential during the beam blips shows no correlation with the 
conditioning status. However, the absolute value measured during the RF phase is noticeably reduced (by around $7 \%$ ) between the first and the last pulse of the performed series. This means that the observed stabilization of the caesium emission (Figure 5b)) and the improved long pulse stability are correlated with a modification of the potential distribution in the plasma volume (during the RF phase of the pulses). This observation has been confirmed during all the following operational days of the ELISE campaign dedicated to long pulse caesium conditioning.

The observed modification of the bias potential during the ongoing conditioning process is correlated with the complex physics of the extended boundary layer: the potential distribution in the plasma is a result of the interplay of charged particle fluxes from the bulk plasma (electrons, positive ions and volume produced negative ions) and the PG surface (surface produced negative ions) with the magnetic filter and the PG bias potential [30]. One possible explanation for the observed decrease of $U_{\text {bias }}$ is the following: since the quasineutrality of the plasma is preserved, even an only slightly increased amount of surface produced negative ions push back a certain amount of electrons towards the bulk plasma [12], resulting in a local decrease of the plasma potential (and thus also of the bias potential). For a comprehensive description of the extended boundary layer physics in caesiated negative hydrogen ion sources the application of full 3D models is necessary. Up to now only first steps towards such a full description have been done $[31,32,33]$.

In the next step the behavior of the plasma during short conditioning pulses is investigated in more detail, taking into account the results of the two Langmuir probes. Figure 6 shows results obtained during one operational day consisting of four long pulses $\left(\mathrm{p}_{\mathrm{fill}}=0.3 \mathrm{~Pa}\right)$ with short pulse $\left(t_{\text {plasma }}=20 \mathrm{~s}, I_{P G}=2.8 \mathrm{kA}, p_{\text {fill }}=0.8 \mathrm{~Pa}\right.$, $P_{R F}=40 \mathrm{~kW} /$ driver) caesium conditioning phases in between. The results of the long pulses cannot be compared among each other (they have been performed at different source parameters) and thus in Figure 6 the results obtained during these pulses are shown in grey.

The bias potential and the plasma potential measured by the Langmuir probes are shown in Figure 6a) and the positive ion density in Figure $6 \mathrm{~b}$ ). Probe measurements have been performed during the RF phase of the pulses and during the beam blip. Shown here are only

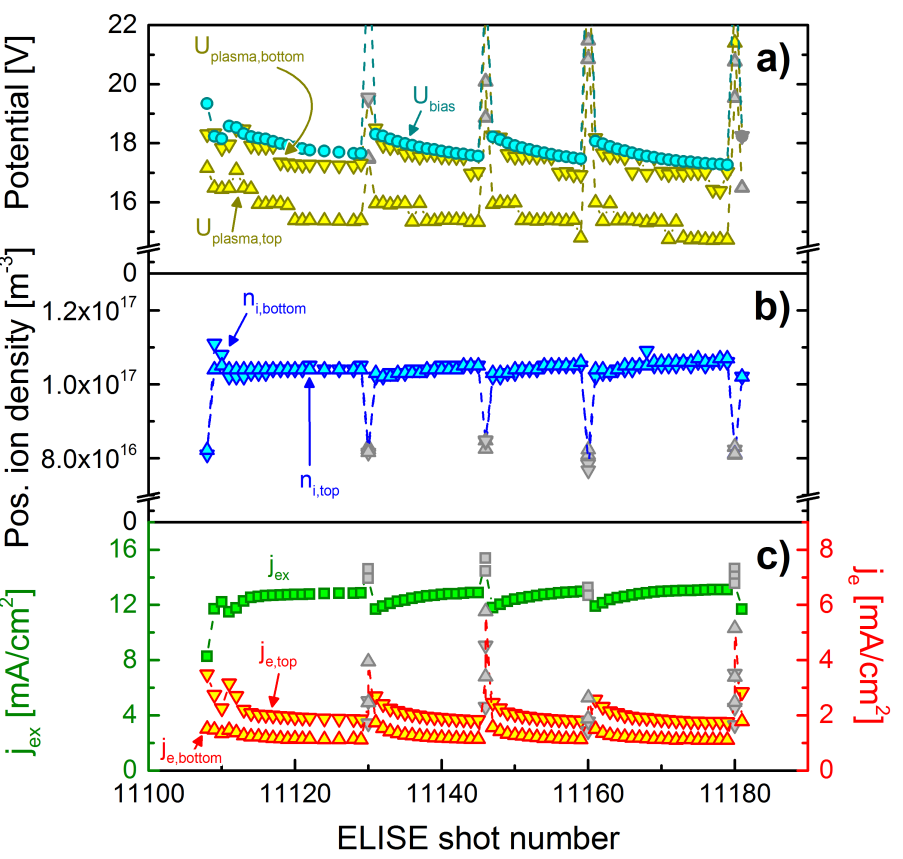

Figure 6: Signals taking during the source conditioning process at $P_{R F}=40 \mathrm{~kW} /$ driver, $p_{\text {fill }}=0.8 \mathrm{~Pa}$ and $I_{P G}=2.8 \mathrm{kA}$. a) Bias potential and plasma potential measured by the two Langmuir probes. Results taken during the long pulses (with different source parameters) are shown in grey. b) Positive ion density measured by the two probes during plasma operation and during the beam blip. c) Extracted the results taken during the RF phase. ion current density and the co-extracted electrons. 
During the short conditioning pulses the bias potential and the plasma potential measured by the top and bottom probe Figure 6a) - show a similar behavior: both potentials decrease from pulse to pulse to a comparable degree. By the long pulses at $p_{\text {fill }}=0.3 \mathrm{~Pa}$ they are pushed upwards in a parallel manner. During the consecutive short conditioning pulses the potentials decrease again, reaching slightly lower values compared to the previous series of conditioning pulses. This observation indicates again that an apparently stable status of the caesium conditioning can be further improved after performing one or more deconditioning pulses. The potential in the lower part of the plasma is by around $2 \mathrm{~V}$ higher compared to the upper part. This observation can be explained by the vertical plasma drift.

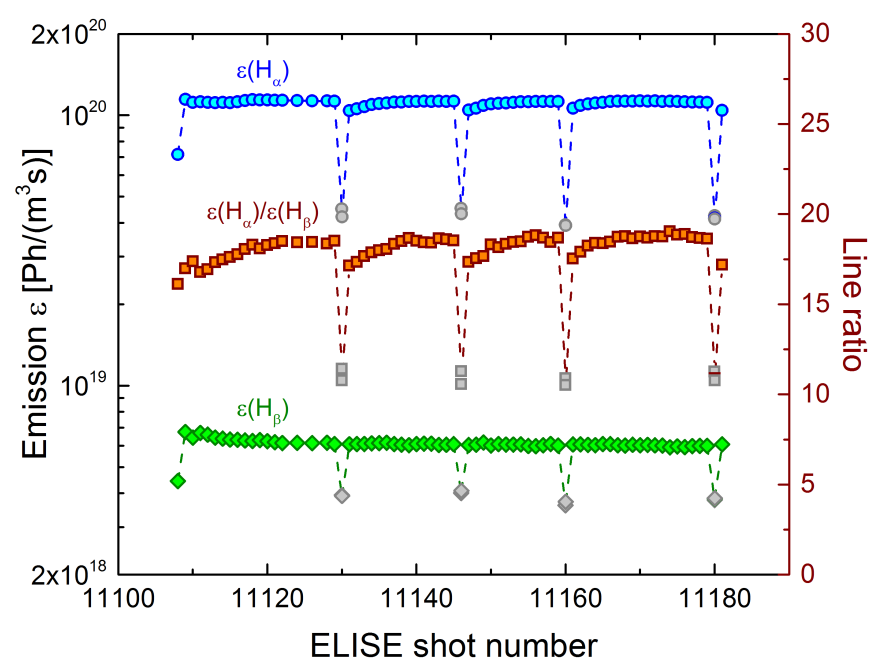

Figure 7: OES signals taking during the source conditioning process at $P_{R F}=40 \mathrm{~kW} /$ driver, $p_{\text {fill }}=0.8 \mathrm{~Pa}$ and $I_{P G}=2.8 \mathrm{kA}$ : emission of the atomic emission lines atomic Balmer emission lines $H_{\alpha}$ and $H_{b}$. Additionally shown is the line ratio $\varepsilon\left(H_{\alpha}\right) / \varepsilon\left(H_{B}\right)$.

This result supports the explanation given above for the decrease of the bias potential (local decrease of the plasma potential caused by an increased number of negative hydrogen ions in the extended boundary layer). Due to the quasi neutrality the electron density is decreased. The extracted negative ion current density increases and the co-extracted electrons are reduced, as shown in Figure 6c).

This hypothesis is supported by OES results, shown in Figure 7: this figure shows the emission of the atomic Balmer emission lines $\mathrm{H}_{\alpha}$ and $\mathrm{H}_{\beta}$ measured along the LOS XLOUu (see Figure 2) as well as the corresponding line ratio $\varepsilon\left(\mathrm{H}_{\alpha}\right) / \varepsilon\left(\mathrm{H}_{\beta}\right)$.

During the short pulse conditioning phases a continuous increase of $\varepsilon\left(\mathrm{H}_{\alpha}\right) / \varepsilon\left(\mathrm{H}_{\beta}\right)$ can be seen. After each of the long deconditioning pulses the $H_{\alpha}$ emission starts again at a significantly lower value while $H_{\beta}$ is almost not affected. Such behaviour can be - for otherwise constant plasma parameters - attributed to an increase of the $\mathrm{H}^{-}$density during the conditioning phases and a subsequent decrease caused by the deconditioning pulse: for the parameters of the extended boundary layer one relevant excitation channel for the Balmer emission is mutual neutralization of $\mathrm{H}^{-}$and $\mathrm{H}^{+}$, ending up predominately in hydrogen atoms in the principal quantum number $n=3$, i.e. the upper level of the $H_{\alpha}$ line [34]. By applying the collisional radiative model for atomic hydrogen based on the Yacora solver [35] it was determined that the observed increase of the $\mathrm{H}_{\alpha} / \mathrm{H}_{\beta}$ line ratio during one of the short pulse conditioning phases can be explained by an increase of the negative hydrogen ion density along the used LOS by around $10 \%$. This value fits very well to the measured increase of the extracted negative ion current density shown in Figure 6c). 
During the conditioning process also the positive ion density - shown in in Figure 6b) - reaches an apparently stable status at the end of each series of short conditioning pulses. After one of the long deconditioning pulses and the following series of conditioning pulses an increased value is reached for the positive ion density. Probably this observation is correlated with an effect predicted by 2D model calculations [36]: in the interplay of surface produced negative ions, electrons from the bulk plasma and the filter field an increased number of negative ions can result in a locally slightly increased plasma density.

The results obtained by the different plasma diagnostics demonstrate the following: the obtained stabilization of the currents extracted during long pulses - mainly the co-extracted electron current obtained by dedicated long pulse conditioning is correlated with only small variations of parameters like the electrostatic potentials and the particle densities and particle fluxes in the extended boundary layer.

\section{Summary and conclusions}

The half-ITER-size NNBI test facility ELISE was used for performing experiments on preparing the ion source - by a dedicated caesium conditioning process - for stable long pulses at a high performance, i.e. a high extracted negative ion current and simultaneously a low co-extracted electron current. The choice of source parameters for such pulses should result from a compromise between high performance and good temporal stability of the extracted currents.

During the caesium conditioning, an apparently stable conditioning status can be further improved by deliberately slightly de-conditioning the source (e.g. by performing one or more long pulse with low filling pressure) and then continuing the conditioning process. The de-conditioning pulse can have an adventurous long-term effect on the conditioning status, resulting in a significant improvement of the long pulse stability - in particular of the co-extracted electrons.

Based on this technique it was possible to perform one-hour-pulses in hydrogen at ITER relevant filling pressure and electron-to-ion ratio at $25 \%$ of the RF power available at ITER per driver, resulting in $25 \%$ of the ITER requirement regarding the extracted negative ion current and an electron-to-ion ratio well below one. However, the stability of pulses gets worse when increasing the RF power, the extraction voltage and when decreasing the strength of the filter field. Thus, for fulfilling the ITER requirements an even better conditioning status is necessary, i.e. the distribution of the caesium reservoirs inside the ion source has to be further improved. Possible measures for reaching such an improved status are - for example-modifying the wall temperatures or the topology of the magnetic filter.

The results of spectroscopic and Langmuir probe measurements demonstrate that the performed long pulse conditioning is correlated with only small variations of parameters like the electrostatic potential, the negative ion density and with the content and temporal behavior of cesium in the extended boundary layer. Fully understanding the complex interplay of the involved physical effects may open the door to the necessary improved conditioning procedures. 


\section{Acknowledgement}

This work has been carried out within the framework of the EUROfusion Consortium and has received funding from the Euratom research and training programme 2014-2018 under grant agreement No 633053. The views and opinions expressed herein do not necessarily reflect those of the European Commission.

The work was supported by a contract from Fusion for Energy (F4E-2009-0PE-32-01), represented by Antonio Masiello. The opinions expressed herein are those of the authors only and do not represent the Fusion for Energy's official position.

\section{References}

[1]: R. Hemsworth, J. Decamps, J. Graceffa, B. Schunke, M. Tanaka, M. Dremel, A. Tanga, H. P. L. De Esch, F. Geli, J. Milnes, T. Inoue, D. Marcuzzi, P. Sonato and P. Zaccaria, Nuclear Fusion 49, 2009, 045006

[2]: B. Schunke, D. Bora, R. Hemsworth and A. Tanga, AIP Conf. Proc. 1097, 2008, 480

[3]: B. Heinemann, H. D. Falter, U. Fantz, P. Franzen, M. Fröschle, R. Gutser, W. Kraus, R. Nocentini, R. Riedl, E. Speth, A. Stäbler, D. Wünderlich, P. Agostinetti and T. Jiang, Fusion Eng. Des. 84, 2009, 915

[4]: P. Franzen, B. Heinemann, U. Fantz, D. Wünderlich, W. Kraus, M. Fröschle, C. Martens, R. Riedl, R. Nocentini, A. Masiello, B. Ruf, L. Schiesko, C. Wimmer and the NNBI-Team, Fusion Eng. Des. 88, 2013, 3132

[5]: A. Masiello, G. Agarici, T. Bonicelli, M. Simon, V. Antoni, H. P. L. De Esch, A. Lorenzi, M. Dremel, P. Franzen, R. Hemsworth, M. Liniers, D. Marcuzzi, D. Martin, R. Piovan, A. Simonin, P. Sonato, E. Surrey, L. Svensson, A. Tanga, V. Toigo, C. Waldon and P. Zaccaria, Fusion Eng. Des. 84, 2009, 1276

[6]: A. Masiello, G. Agarici, T. Bonicelli, F. Fantini, M. Gagliardi, M. Paolucci, M. Simon, P. Wikus, P. Agostinetti, M. Bigi, P. Blatchford, D. Boilson, M. Dalla Palma, C. Day, A. De Lorenzi, M. Dremel, H. Decamps, P. Franzen, J. Graceffa, B. Heinemann, S. Hanke, R. Hemsworth, M. Kuriyama, A. Luchetta, D. Marcuzzi, M. Pavei, R. Pearce, N. Pilan, W. Rigato, B. Schunke, P. Sonato, L. Svensson, P. Thomas, V. Toigo and P. Zaccaria, in Proc. 24th IAEA Fusion Energy Conference, San Diego, USA, 2012, http://wwwnaweb.iaea.org/napc/physics/FEC/FEC2012/html/fec12.htm.

[7]: E. Speth, H. D. Falter, P. Franzen, U. Fantz, M. Bandyopadhyay, S. Christ, A. Encheva, M. Fröschle, D. Holtum, B. Heinemann, W. Kraus, A. Lorenz, C. Martens, P. McNeely, S. Obermayer, R. Riedl, R. Süss, A. Tanga, R. Wilhelm and D. Wünderlich, Nucl. Fusion 46, 2006, S220

[8]: R. Hemsworth, A. Tanga and V. Antoni, Rev. Sci. Instrum. 79, 2008, 02 C109

[9]: P. Sonato, P. Agostinetti, G. Anaclerio, V. Antoni, O. Barana, M. Bigi, M. Boldrin, M. Cavenago, S. Dal Bello, M. Dalla Palma, A. Daniele, M. D'Arienzo, A. Lorenzi, A. Ferro, A. Fiorentin, E. Gaio, E. Gazza, L. Grando, F. Fantini, F. Fellin, A. Luchetta, G. Manduchi, F. Milani, D. Marcuzzi, L. Novello, R. Pasqualotto, M. Pavei, R. Pengo, S. Peruzzo, A. Pesce, N. Pilan, R. Piovan, N. Pomaro, M. Recchia, W. Rigato, A. 
Rizzolo, G. Serianni, M. Spolaore, P. Spolaore, S. Sandri, C. Taliercio, V. Toigo, M. Valisa, P. Veltri, P. Zaccaria, A. Zamengo and L. Zanotto, Fusion Eng. Des. 84, 2009, 269

[10]: P. Sonato, D. Boilson, T. Bonicelli, A. K. Chakraborty, C. Day, P. Franzen, G. Gorini, T. Inoue, J. Milnes, T. Minea, H. P. L. De Esch, P. Agostinetti, M. Agostini, V. Antoni, M. Barbisan, P. Bettini, M. Bigi, M. Boldrin, M. Brombin, M. Cavenago, G. Chitarin, G. Croci, M. Dalla Palma, S. Dal Bello, M. De Muri, A. De Lorenzi, F. Fellin, A. Ferro, A. Fiorentin, L. Grando, S. Hanke, M. Kashiwagi, A. Luchetta, G. Manduchi, N. Marconato, D. Marcuzzi, R. Pasqualotto, M. Pavei, S. Peruzzo, A. Pesce, N. Pilan, N. Pomaro, M. Recchia, W. Rigato, A. Rizzolo, E. Sartori, G. Serianni, A. Soppelsa, M. Sottocornola, M. Spolaore, C. Taliercio, M. Taniguchi, H. Tobari, V. Toigo, M. Valente, P. Veltri, K. Wanatabe, P. Zaccaria, B. Zaniol, A. Zamengo and L. Zanotto, in Proc. 24th IAEA Fusion Energy Conference, San Diego, USA, 2012. http://www-naweb.iaea.org/napc/physics/FEC/FEC2012/html/fec12.htm.

[11]: M. Fröschle, U. Fantz, P. Franzen, W. Kraus, R. Nocentini, L. Schiesko, D. Wünderlich and the NNBITeam, Fusion Eng. Des. 88, 2013, 1015

[12]: U. Fantz, P. Franzen, W. Kraus, M. Berger, S. Christ-Koch, M. Fröschle, R. Gutser, B. Heinemann, C. Martens, P. McNeely, R. Riedl, E. Speth and D. Wünderlich, Plasma Phys. Control. Fusion 49, 2007, B563

[13]: M. Bacal, Nucl. Fusion 46, 2006, S250

[14]: B.S. Lee, M. Seidl, Appl. Phys. Lett. 61, 1992, 2857

[15]: R. Gutser, C. Wimmer and U. Fantz, Rev. Sci. Instrum. 82, 2011, 023506

[16]: P. Franzen, L. Schiesko, M. Fröschle, D. Wünderlich, U. Fantz and the NNBI-Team, Plasma Phys. Control. Fusion 53, 2011, 115006

[17]: M. Fröschle, R. Riedl, H. D. Falter, R. Gutser, U. Fantz and the NNBI-Team, Fusion Eng. Des. 84, 2009, 788

[18]: R. Gutser, D. Wünderlich, U. Fantz and the NNBI-Team, Plasma Phys. Control. Fusion 53, 2011, 105014

[19]: U. Fantz, P. Franzen, W. Kraus, M. Berger, S. Christ-Koch, H. D. Falter, M. Fröschle, R. Gutser, B. Heinemann, C. Martens, P. McNeely, R. Riedl, E. Speth, A. Stäbler and D. Wünderlich, Nucl. Fusion 49, 2009,125007

[20]: U. Fantz, C. Wimmer, Rev. Sci. Instrum. 83, 2012, 02B110

[21]: W. Kraus, U. Fantz, P. Franzen, M. Fröschle, B. Heinemann, R. Riedl and D. Wünderlich, Rev. Sci. Instrum. 83, 2012, 02B104

[22]: P. Franzen, U. Fantz, D. Wünderlich, B. Heinemann, R. Riedl, W. Kraus, M. Fröschle, B. Ruf, R. Nocentini and the NNBI-Team, Nucl. Fusion 55, 2015, 053005 
[23]: P. Franzen, D. Wünderlich, U. Fantz and the NNBI-Team, Plasma Phys. Control. Fusion 56, 2014, 025007

[24]: R. Nocentini, U. Fantz, P. Franzen, M. Fröschle, B. Heinemann, R. Riedl, B. Ruf, D. Wünderlich and the NNBI-Team, Fusion Eng. Des. 88, 2013, 913

[25]: D. Wünderlich, U. Fantz, P. Franzen, R. Riedl and F. Bonomo, Rev. Sci. Instrum. 84, 2013, 093102

[26]: U. Fantz, H. D. Falter, P. Franzen, D. Wünderlich, M. Berger, A. Lorenz, W. Kraus, P. McNeely, R. Riedl and E. Speth, Nucl. Fusion 46, 2006, S297

[27]: U. Fantz, H. D. Falter, P. Franzen, M. Bandyopadhyay, B. Heinemann, W. Kraus, P. McNeely, R. Riedl, E. Speth, A. Tanga and R. Wilhelm, Fusion Eng. Des. 74, 2005, 299

[28]: P. Franzen, U. Fantz, W. Kraus, M. Berger, S. Christ-Koch, M. Fröschle, R. Gutser, B. Heinemann, C. Martens, F. Maisberger, P. McNeely, R. Riedl, E. Speth, D. Wünderlich and T. Zacharias, AIP Conf. Proc. $993,2008,51$

[29]: W. Kraus, M. Berger, U. Fantz, P. Franzen, M. Fröschle, B. Heinemann, R. Riedl, E. Speth, A. Stäbler and D. Wünderlich, AIP Conf. Proc. 1097, 2009, 275

[30]: U. Fantz, P. Franzen and D. Wünderlich, Chem. Phys. 398, 2012, 7

[31]: S. Mochalskyy, A.F. Lifschitz and T. Minea, J. Appl. Phys. 111, 2012, 113303

[32]: F. Taccogna, P. Minelli and S. Longo, Plasma Sources Sci. Technol. 22, 2013, 045019

[33]: G. Fubiani, J.P. Boeuf, Phys. Plasmas 21, 2014, 073512

[34]: U. Fantz, D. Wünderlich, New J. Phys. 8, 2006, 301

[35]: D. Wünderlich, S. Dietrich and U. Fantz, J. Quant. Spectrosc. Radiat. Transfer 110, 2009, 62

[36]: T. Sakurabayashi, A. Hatayama and M. Bacal, J. Appl. Phys. 95, 2004, 3937 